This is a pre-publication version of a paper that will appear as Smart, P. R. (2013) Embodiment, Cognition and the World Wide Web. In L. Shapiro (Ed.), The Routledge Handbook of Embodied Cognition. Routledge, New York, New York, USA (http://www.amazon.co.uk/The-RoutledgeHandbook-Embodied-Cognition/dp/0415623618/ref=sr 1 1?ie=UTF8\&qid=1360501631\&sr=8-1).

\title{
Embodiment, Cognition and the World Wide Web
}

Paul R. Smart

School of Electronics and Computer Science, University of Southampton, Southampton, SO17 1BJ, UK.

\section{Introduction}

Embodied cognition forms part of an increasingly popular trend in the philosophy of mind and cognitive science which emphasizes the role played by extra-neural and extra-corporeal factors in human cognitive processing (Clark, 2008; Shapiro, 2011). It sits alongside a number of other areas of research, which we can collectively refer to as embodied, embedded, enactive and extended (4E) approaches to cognition. Although subtle differences exist between these approaches, what they have in common is a commitment to the idea that issues of material embodiment and environmental embedding play explanatorily significant roles in our understanding of human cognitive success.

The emphasis that $4 \mathrm{E}$ approaches place on extra-neural and extra-corporeal factors suggests that we should pay close attention to the various contexts in which cognition occurs. In this respect, the advent of the World Wide Web is of significant interest. The Web has rapidly emerged to transform the nature of many of our daily activities, and its growing popularity, in conjunction with the increasing ubiquity of Web-enabled devices and wireless networks, means that the Web now forms an increasingly prevalent part of the broader environmental context in which much of our daily thought and action is situated. Inasmuch as our cognitive profiles are shaped by information processing loops that extend beyond the traditional biological borders of skin and skull, it is natural to wonder what effect this new form of technology-mediated information access will have on our individual cognitive profiles. If, as Clark (2007) suggests, we are 'profoundly embodied agents', ones capable of "incorporating new resources and structures deep into their problem-solving engines" (pg. 277), then it seems at least possible that the Web may serve as the target of future forms of biotechnological merger, perhaps even extending the machinery of the mind to encompass aspects of the online world (see Smart, 2012).

In addition to this potential transformation of our individual cognitive profiles, the Web also affords new opportunities for social interaction and engagement, made possible by an ever-expanding array of social media sites and social networking applications. We have, as yet, little understanding of how these new technologies will affect the social aspects of human cognition, both in terms of our ability to process social information as well as our ability to distribute cognitive processes across groups of individuals. Again, this seems to constitute an important area of attention for the $4 \mathrm{E}$ theorist. Sociocultural factors often surface in $4 E$ discussions of human cognition (e.g., Hutchins, 2008), and the social aspects of cognition are an increasingly important focus of theoretical and research attention in the embodied mind literature (Smith, 2008). 
In this chapter, I attempt to provide an overview of some of the issues that are raised when looking at the Web through the lens of $4 E$ approaches to cognition. I first discuss the way in which emerging forms of interaction with the Web may lay the basis for future forms of Web-based cognitive extension (Smart, 2012). I then go on to discuss some of the issues surrounding what might be called the 'Social Web' - the part of the Web that is concerned with the enablement of social interactions, social relationships and collaborative enterprises.

\section{Embodiment and the Real World Web}

At first sight, a discussion of the Web may seem somewhat out of place in a text on embodied cognition. Work within embodied cognition (and $4 \mathrm{E}$ cognition, in general) tends to focus on situations in which we are actively engaged with the real world, exploiting all manner of sensorimotor cues in order to realize intelligent thought and action. The nature of our interaction with the Web seems far removed from this sort of situation. Even though we might be justified in seeing the Web as an important part of context in which cognition occurs - part of the material backdrop against which our thoughts and actions take shape - it is by no means clear that the details of our physical embodiment and environmental embedding really matter that much when it comes to understanding the nature of our online interactions.

One response to the claim that Web-based interactions are not a legitimate point of interest for the embodied cognition theorist is to downplay the distinction between the notions of online and offline behaviour. Thus it might be argued that our interaction with the Web is, in fact, a genuine case of embodied interaction which surely does take place in the real world. The nature of the sensorimotor dynamic might be different from that seen in the case of (e.g.) running to catch a flyball (see McBeath et al., 1995), but it is far from clear that all the details of material embodiment and environmental embedding are irrelevant to understanding our life online. It might also be said, of course, that an increasing amount of cognition takes place online - that the Web is a forum in which we confront some of our most potent intellectual and cognitive challenges. It would be disappointing, indeed, if the $4 \mathrm{E}$ theorist had nothing to say about such matters.

Another response to the challenge that the Web falls outside the realms of interest for the embodied cognition theorist is to deny that the traditional notions of online and offline interaction are of any real relevance when it comes to contemporary forms of interaction and engagement with the Web. Our predominant vision of online interaction is one in which we are sat in front of a desktop computer, accessing the Web through a conventional browser-based interface (such as Internet Explorer or Google Chrome). In these cases, we are encouraged to see the flow of our thoughts as somewhat decoupled from the 'real world', as occurring in response to remotely located information resources and as being largely unaffected by events in the sensory periphery of the computer screen. The advent of mobile and portable computing solutions is, of course, changing all this. Increasingly, our interactions with the Web are ones that take place in the context of our everyday sensorimotor engagements with the world, where our attention constantly switches from the Web to the wider world according to the demands of the tasks in which we are engaged. Consider, for example, the case of a person equipped with an iPhone and located in an unfamiliar city. Suppose this person's knowledge of the city in question is limited, but they wish to walk to a nearby location. We now see them engaged in a behaviour where the act of walking to the location is guided by the feedback they receive from their GPS- and Web-enabled device. For the most part, the individual's attention is focused on aspects of the physical environment (cars, people, 
pushchairs, and various other obstacles). However, at critical junctures in the journey, attention adverts to the iPhone and information is retrieved in order to inform decisions about which direction to take. How should we view the online/offline status of the person in this case? Are they online, offline, or some mixture of the two? It is in cases like these, I suggest, where the emerging panoply of portable devices and modes of practice concerning Web usage lead to an effective blurring of our notions about what constitutes online and offline behaviour. The new devices enable us to interleave our interactions with the Web and the real world in a way that makes the traditional distinction between offline and online interaction of nugatory significance. As Floridi (2011) suggests, our lives are increasingly complex mergers of online and offline interaction - our lives are increasingly led 'onlife'.

The growing trend in the use of mobile and portable computing solutions marks an important shift in the way in which we access the Web, and it is one that opens up a range of opportunities for us to exploit Web-based content as part of our everyday embodied interactions with the world. The trend is likely to continue in the near future with the advent of wearable computing solutions, such as the head-mounted augmented reality display device envisioned by Google's Project Glass initiative. These sorts of devices promise to transform the nature of our contact with the Web, making information directly available within the visual field and thereby reducing the need to switch our attention between a technological resource (e.g., an iPhone) and the real world. In addition, such devices promise to reduce the demand placed on our bodies to manage information retrieval operations. In the case of Google's Project Glass, for example, it has been suggested that the device will be sensitive to natural language voice commands, thereby enabling hands-free modes of operation. These kinds of interaction are important because they liberate bodily resources to engage in other kinds of activities, some of which may be essential to embodied cognitive processes. Crucially, from an embodied cognition perspective, hands-free modes of operation allow the user to engage in gestures, and these have been shown to play a role in enhancing various aspects of human cognitive processing (Goldin-Meadow, 2003; McNeill, 2005).

Another device that is of interest in the current context is the Web-enabled umbrella described by Matsumoto et al (2008). The umbrella features a variety of sensors (e.g., GPS, compass, accelerometer, etc.), and it is able to project Web-based content directly into the user's field of view by virtue of a projection device focused on the underside of the umbrella canopy. By providing the user with a range of interaction opportunities (e.g., the normal turning, dipping, and twisting actions that people perform with umbrellas), and by also integrating information from a variety of sensors and Web services, the umbrella is able to present a variety of forms of context-relevant information that take into account both the user's physical location, as well as their current interests and activities. Interestingly, and of particular relevance in the current context, Matsumoto et al (2008) describe their work as part of an effort to realize what they call the 'Embodied Web': a form of enhanced interactivity in which "natural embodied interactions...augments [a users] experience in the real world" (pg. 49).

The work of researchers like Matsumoto et al (2008), as well as the research and development efforts of organizations like Google, will, in all likelihood, transform the nature of our future interaction with the Web. In place of conventional forms of browser-based access, we are witnessing the transition to an era in which the Web is placed at the heart of our everyday embodied interactions with the world. We may view the end state of this transition as resulting in what has 
been called the 'Real World Web' (Smart, 2012), a vision of the Web in which we encounter increasingly intimate forms of sensorimotor contact with Web-based information. The realization of this vision will serve to blur the distinction between online and offline behaviour and enable us to see the Web as just another part of what Hutchins (2010) calls the 'cognitive ecology' - the set of neural, bodily, social, technological and environmental factors that together shape the course of our cognitive endeavours.

\section{The Web-Extended Mind}

Devices that increase both the accessibility and perceptual availability of Web-based information have a number of implications for how we view the potential cognitive impact of the Web. One such implication concerns the possibility for Web-based forms of cognitive extension in which the technological and informational elements of the Web come to form part of the supervenience base for (at least some) mental states and processes. As part of their seminal paper on the extended mind, Clark and Chalmers (1998) outlined a thought experiment in which a neurologically-impaired individual, Otto, relied on the use of an external resource (a notebook) in order to achieve certain tasks. The main point of the thought experiment was to highlight the similar functional role played by both biological (i.e., the brain/body) and non-biological (e.g., the notebook) resources in supporting at least some cases of intentional action. Inasmuch as the bio-external resources played a role similar to that served by biological resources, Clark and Chalmers claimed, we should view their contributions to global behaviour as on a functional par. This would, at least in some cases, enable us to see bio-external resources as playing a constitutive role in the realization of mental states and processes. When we apply such notions to the Web, we can entertain the possibility of Webextended minds, or minds in which the technological and informational elements of the Web come to be seen as part of the physical machinery of a bio-technologically hybrid cognitive system (see Smart, 2012).

In order to outline the notion of the Web-extended mind, we can adapt the example provided by Clark and Chalmers, dispensing with the technologically low-grade notebook and replacing it with technologies that support sophisticated forms of Web access (in the manner alluded to by the vision of the Real World Web). Let us therefore imagine a human agent who is equipped with a mobile networked device (a mobile phone will do) in order to provide wireless access to the Web, an augmented reality head-mounted display device (similar to the technological target envisioned by Google's Project Glass initiative), and a means of controlling information retrieval (for the sake of argument, imagine something along the lines of the electromyographic, electroencephalographic and electrooculographic interfaces currently being developed by a variety of academic and commercial organizations (Mason et al., 2007; Nicolelis, 2001)). Thus equipped, our subject is able to retrieve information from the Web, on demand, in a manner that is able to shape the course of their thoughts and actions in a number of task contexts. Our subject could, for example, be guided to the location of interesting spatial targets by the use of simple geo-registered directional indicators overlaid onto the visual field. Our subject would not, therefore, have to rely on bio-memory to recall facts, such as the location of particular places of interest, because location-aware services would retrieve and present this information in a way that would serve to guide ongoing behaviour. Similarly, imagine that our subject has an interest in baseball and that baseball facts and figures are continually posted on the Web in a form that permits flexible forms of retrieval, combination, aggregation and inference (for example, the data might be available in the form of a linked data 
resource (see Heath \& Bizer, 2011)). In this situation, our subject would be able to retrieve any piece of baseball-related information, on demand, in a manner that is robustly and continuously available. What would our impressions be about the subject's epistemic capabilities in this situation? Would it be appropriate for us to say that the subject pretty much 'knows' everything there is to know about baseball, at least in terms of the information that is posted on the Web? One reason to suspect that this might be the case is that what seems to determine whether we know or do not know something is not the fact that we are continuously, consciously aware of relevant facts and figures; rather, what seems to count is more the kind of access we have to relevant information. If our access to externally-located information was just as reliably, easily and continuously available as the kind of access afforded by our own bio-memories, then we can question whether there is any principled reason to insist that the external information would not count as part of our own personal body of knowledge and (dispositional) beliefs about the world (see Clark, 2003; pg. 42).

\section{The Social Web}

In addition to its potential effects on our individual cognitive profile, the Web also plays an important role in socially-situated cognition. Ever since the advent of Web 2.0, which is characterized by greater levels of user participation in the creation, maintenance and editing of online content, the Web has provided ample opportunities to support various forms of sociallydistributed information processing. In addition, the recent surge in social media sites (e.g., YouTube), social networking systems (e.g., Facebook) and microblogging services (e.g., Twitter) has opened up new ways for people to interact, communicate and share information content. We are increasingly seeing the emergence of what we might call the 'Social Web': a suite of applications, services, technologies, formats, protocols and other resources, all united in their attempt to both foster and support social interaction.

Perhaps unsurprisingly, the Social Web is of considerable interest to those who approach cognition from a $4 \mathrm{E}$ perspective. This is because human cognition is often seen as a socio-culturally situated activity, and great emphasis is placed on the role of social forces and factors in shaping our cognitive capabilities. One point of interest here for the $4 \mathrm{E}$ theorist concerns the nature of socially-derived information on the Web and the way in which this information influences the processes associated with person perception and social sensemaking (i.e., the attempt to understand the behaviour of others in terms of intentional mental states). Research in this area has revealed that cues derived from the online environment (e.g., information about a person's social network) can be used to drive social judgements relating to (e.g.) credibility, extraversion, popularity and social attractiveness (Tong et al., 2008; Westerman et al., 2012). Such findings highlight an issue of critical importance when it comes to the Web's role in supporting social cognition. This is the fact that many of the cues available in the online realm are ones that could not be (easily) accessed in conventional face-to-face contexts. Thus, rather than see Web-based forms of social interaction as an inherently weaker or impoverished version of that which takes place in conventional face-to-face encounters (at least from the perspective of social cognition), we are encouraged to view the Web as an environment that surpasses many of the constraints associated with co-presence and co-location. By situating social interaction on the Web, we are presented with a range of opportunities to influence social cognition, and many of these opportunities are simply out of reach in face-to-face social exchanges.

Another point of interest for the $4 \mathrm{E}$ theorist concerns the way in which some cognitive processes, such as reasoning, remembering and problem-solving, might be seen as distributed across a group or 
team of individuals (Hutchins, 1991, 1995). Within the context of the Web and Internet Science community, the advent of the Social Web has given rise to an increasing interest in the sociallydistributed nature of human cognition (Chi, 2008, 2009; Kearns, 2012), and this interest has been accentuated with the recent explosion in social computing (Parameswaran \& Whinston, 2007), human computation (Quinn \& Bederson, 2011) and collective intelligence (Bonabeau, 2009) systems. Such technologies focus attention on the ways in which the Web may be used to exploit the latent 'socio-cognitive capital' possessed by large numbers of physically-distributed individuals.

Because of the kinds of opportunities it affords for large-scale collaboration, information sharing, and the coordination of collective efforts, the Web emerges as a seemingly natural platform to realize advanced forms of collective intelligence. However, in spite of the apparent potential of the Web to support socially-distributed cognition, it is important to understand that not all forms of Web-based social interaction and information exchange necessarily lead to improvements in collective cognitive processing. It is known, for example, that the rate at which information and ideas are distributed through a social network can have a profound effect on group-level cognitive outcomes, and this highlights a source of tension in our attempts to engineer systems that support socially-distributed cognition in Web-based contexts. On the one hand, we are usually inclined to countenance high-bandwidth communication systems that feature high levels of connectivity and which maximize the efficient and widespread dissemination of information to all members of a community. On the other hand, we encounter a range of findings in the social psychological and multi-agent simulation literature that suggest that such systems may not always deliver the best outcomes in terms of collective cognitive performance. In some situations, at least, the rapid communication of information and ideas does not always serve the collective cognitive good: precipitant forms of information sharing can sometimes subvert rather than support sociallydistributed cognition (Hutchins, 1991; Lazer \& Friedman, 2007).

What all this means, of course, in terms of our attempt to support socially-distributed cognition on the Web, is that we need to develop a better understanding of the effect that different forms of information flow and influence have on collective cognitive outcomes. One factor that has emerged as an important focus of research attention, in this respect, is the structure of the communication network in which individuals are embedded. Research has shown that the structure of the communication network shapes the flow of information between individuals, and this can lead to different effects on group-level performance. Interestingly, the suitability of different forms of network structure seems to depend on the nature of the task that is being performed (Kearns, 2012). Thus, when subjects are confronted with a simple problem, it seems that the best structure is one that connects all individuals to every other individual in the group (i.e., a fully-connected network) (Lazer \& Friedman, 2007; Smart et al., 2010). On more complex problems, however, it seems that more limited forms of connectivity are desirable because such networks essentially impede the rate of information flow between the individuals and thus prevent premature convergence on sub-optimal or inaccurate solutions (Lazer \& Friedman, 2007; Smart et al., 2010).

Another factor that has proved of considerable research interest concerns the amount of feedback that is given to individuals about the progress or status of collective cognitive processing. This is of particular interest in a Web-based context since the Web provides a unique opportunity to gather and exploit information about the judgements, decisions or activities of individual participants. Consider, for example, the attempt by a group of users to derive an estimate of some unknown 
parameter. For the sake of argument, let us say that participants are asked to estimate the number of crimes that are recorded in the city of London. In this situation, the statistical average of everyone's estimates should approximate the actual number of crimes recorded, and this exemplifies one way in which a system like the Web may be used to exploit what has become known as the Wisdom of Crowds (Surowiecki, 2005). Imagine, however, that a Web-based system that mediates this instance of collective intelligence provides feedback on the estimates that have been made by users on previous occasions. How will the provision of this information influence the ratings of new users? And, in the long-term, will the feedback lead to better or worse performance relative to what might be expected in situations where no feedback is given at all?

In order to answer these questions, Lorenz et al (2011) devised an experiment in which participants were asked to generate ratings in response to a number of questions - the answers to which were not known in advance by any one individual. They then manipulated the level of feedback that participants were given about the responses of other participants across a number of trials. Their results reveal that feedback often works to undermine collective performance. Rather than being able to derive estimates that were, at the collective level, close to the actual answer, subjects in the high feedback condition settled on responses that were, at the collective level, worse than those seen in situations where subjects received no feedback at all. In accounting for their results, Lorenz et al (2011) posit a 'social influence effect' in which the feedback about other users' ratings is deemed to progressively reduce the diversity of ratings within the group without a corresponding improvement in group-level accuracy. These results suggest that although the Web provides an environment in which a variety of kinds of information can be gathered during the course of sociallydistributed information processing, not all of this information should be made available to the individual agents engaged in the process. Instead, the results call for a more nuanced approach in which the system works to adaptively regulate the availability of different kinds of information in ways that are sensitive to the nature of the task that is being performed, as well as the psychological propensities of the participating agents. In essence, what is required is a way of dynamically organizing the setup of Web-based socio-technical systems in order to meliorate group-level cognitive processes in a variety of different task contexts.

\section{Conclusion}

A key feature of the embodied cognition perspective is the emphasis it places on extra-neural factors in accounting for our human cognitive success. In particular, the embodied cognition perspective emphasizes the fact that the human brain is an extremely plastic, profoundly embodied and socioculturally situated organ. Rather than see intelligence as something that is located in a purely inner, neural realm, embodied cognition emphasizes the way in which cognition depends on forces and factors that are distributed across the brain, the body and the world. This emphasis makes the World Wide Web of considerable interest to the embodied cognition theorist; for the Web is an increasingly prevalent part of the wider cognitive ecology in which some of our most intellectually and cognitively challenging endeavours are situated. A number of emerging technologies seem poised to place the Web at the heart of our everyday sensorimotor interactions with the world, and inasmuch as we are profoundly embodied agents, constantly engaged in the re-negotiation of our bodily and cognitive frontiers (see Clark, 2007), the Web seems to provide a range of opportunities for the deep and transformative restructuring of our cognitive capabilities. 
The Web is also a platform for social interaction and engagement, and this opens up the possibility that the Web may lead to new forms of socially-situated and socially-distributed cognition. One point of interest concerns the way in which the Web can support forms of social cognition associated with person perception and social understanding. Recent research is suggesting that by situating social interactions on the Web, we are provided with a range of opportunities to influence social cognition, and these opportunities are often out of reach in conventional face-to-face contexts. Similarly, when it comes to socially-distributed cognition, the Web provides a platform in which we have a relatively unique opportunity to organize information flows in ways that fully exploit the socio-cognitive capital of geographically-dispersed individuals.

\section{Acknowledgements}

This research was sponsored by the U.S. Army Research Laboratory and the U.K. Ministry of Defence and was accomplished under Agreement Number W911NF-06-3-0001. The views and conclusions contained in this document are those of the author(s) and should not be interpreted as representing the official policies, either expressed or implied, of the U.S. Army Research Laboratory, the U.S. Government, the U.K. Ministry of Defence or the U.K. Government. The U.S. and U.K. Governments are authorized to reproduce and distribute reprints for Government purposes notwithstanding any copyright notation hereon.

\section{References}

Bonabeau, E. (2009) Decisions 2.0: The Power of Collective Intelligence. MIT Sloan Management Review, 50(2), 45-52.

Chi, E. H. (2008) The Social Web: Research and Opportunities. Computer, 41(9), 88-91.

Chi, E. H. (2009) Augmented social cognition: using social web technology to enhance the ability of groups to remember, think, and reason. 35th SIGMOD International Conference on Management of Data Providence, Rhode Island, USA.

Clark, A. (2003) Natural-Born Cyborgs: Minds, Technologies and the Future of Human Intelligence. Oxford University Press, Oxford, UK.

Clark, A. (2007) Re-Inventing Ourselves: The Plasticity of Embodiment, Sensing, and Mind. Journal of Medicine and Philosophy, 32(3), 263-282.

Clark, A. (2008) Supersizing the Mind: Embodiment, Action, and Cognitive Extension. Oxford University Press, New York, USA.

Clark, A., \& Chalmers, D. (1998) The Extended Mind. Analysis, 58(1), 7-19.

Floridi, L. (2011) The Construction of Personal Identities Online. Minds and Machines, 21(4), 477479.

Goldin-Meadow, S. (2003) Hearing Gesture: How Our Hands Help Us Think. Harvard University Press, Cambridge, Massachusetts, USA.

Heath, T., \& Bizer, C. (2011) Linked Data: Evolving the Web into a Global Data Space (1st edition ed.). Morgan \& Claypool

Hutchins, E. (1991) The social organization of distributed cognition. In L. Resnick, J. Levine \& S. Teasley (Eds.), Perspectives on Socially Shared Cognition. The American Psychological Association, Washington DC, USA.

Hutchins, E. (1995) Cognition in the Wild. MIT Press, Cambridge, Massachusetts, USA.

Hutchins, E. (2008) The role of cultural practices in the emergence of modern human intelligence. Philosophical Transactions of the Royal Society B: Biological Sciences, 363(1499), 2011-2019.

Hutchins, E. (2010) Cognitive ecology. Topics in Cognitive Science, 2(4), 705-715.

Kearns, M. (2012) Experiments in social computation. Communications of the ACM, 55(10), 56-67.

Lazer, D., \& Friedman, A. (2007) The Network Structure of Exploration and Exploitation. Administrative Science Quarterly, 52(4), 667-694. 
Lorenz, J., Rauhut, H., Schweitzer, F., \& Helbing, D. (2011) How social influence can undermine the wisdom of crowd effect. Proceedings of the National Academy of Sciences, 108(22), 90209025.

Mason, S., Bashashati, A., Fatourechi, M., Navarro, K., \& Birch, G. (2007) A comprehensive survey of brain interface technology designs. Annals of Biomedical Engineering, 35(2), 137-169.

Matsumoto, T., Hashimoto, S., \& Okude, N. (2008) The embodied Web: Embodied Web-services interaction with an umbrella for augmented city experiences. Computer Animation and Virtual Worlds, 19(1), 49-66.

McBeath, M. K., Shaffer, D. M., \& Kaiser, M. K. (1995) How baseball outfielders determine where to run to catch fly balls. Science, 268(5210), 569-573.

McNeill, D. (2005) Gesture and Thought. University of Chicago Press, Chicago, USA.

Nicolelis, M. (2001) Actions from thoughts. Nature, 409(6818), 403-407.

Parameswaran, M., \& Whinston, A. B. (2007) Research issues in social computing. Journal of the Association for Information Systems, 8(6), 336-350.

Quinn, A. J., \& Bederson, B. B. (2011) Human computation: a survey and taxonomy of a growing field. Annual Conference on Human Factors in Computing Systems (CHI'11), Vancouver, British Columbia, Canada.

Shapiro, L. A. (2011) Embodied cognition. Routledge, Abingdon, Oxfordshire, UK.

Smart, P. R. (2012) The Web-Extended Mind. Metaphilosophy, 43(4), 426-445.

Smart, P. R., Huynh, T. D., Braines, D., \& Shadbolt, N. R. (2010) Dynamic Networks and Distributed Problem-Solving. Knowledge Systems for Coalition Operations (KSCO'10), Vancouver, British Columbia, Canada.

Smith, E. R. (2008) Social relationships and groups: New insights on embodied and distributed cognition. Cognitive Systems Research, 9(1), 24-32.

Surowiecki, J. (2005) The Wisdom of Crowds. Random House, New York, USA.

Tong, S. T., Van Der Heide, B., Langwell, L., \& Walther, J. B. (2008) Too much of a good thing? The relationship between number of friends and interpersonal impressions on Facebook. Journal of Computer-Mediated Communication, 13(3), 531-549.

Westerman, D., Spence, P. R., \& Van Der Heide, B. (2012) A social network as information: The effect of system generated reports of connectedness on credibility on Twitter. Computers in Human Behavior, 28, 199-206. 